\title{
PLANETARY NEBULA FORMATION
}

\author{
SUN KWOK \\ Department of Physics and Astronomy \\ University of Calgary \\ Calgary, Alberta, Canada T2N $1 N 4$
}

\section{Introduction}

The idea that planetary nebulae (PN) originate from the outer atmospheres of red giant was first proposed by Shkloskii (1956). In the early 1970's, it was generally believed that PN were created by a sudden envelope ejection; however, the exact nature of the instability responsible for the ejection was not understood. Furthermore, it was not clear how the observed masses of PN are related to the amount of mass ejected, and there was no explanation of how the observed shell morphologies of PN could be created by such instabilities. From the observed sizes and expansion velocities of PN, we know that the dynamical lifetime of a $\mathrm{PN}$ is $\sim 10^{4} \mathrm{yr}$. During this period, the star has to evolve from the red side of the H-R diagram, where the envelope is ejected, to the blue side of the H-R diagram, where the stellar temperature is high enough to photoionize the ejecta. If the star evolves too slowly, the nebula will have dispersed into the interstellar medium before it is ionized. If the star evolves too fast, then the nebula will only be illuminated for a short time before the stellar luminosity drops on the white dwarf cooling track (Renzini 1982). It is therefore surprising that PN is such a common phenomenon, with $\sim 30000$ PN estimated to be in the Galaxy now.

If we do not accept that the existence of PN is a fortuitous coincidence, then we have seek a physical explanation to the formation of PN that will bring about a synchronous evolution of the nebula and the central star. Furthermore, such a physical process should also explain the observed morphology and physical properties (density, velocity, etc) of PN.

\section{Progenitors of planetary nebulae: asymptotic giant branch stars}

Beginning in the late 1960's, infrared and millimetre line observations revealed that all late asymptotic giant branch (AGB) stars are losing mass via a stellar wind. The rate of mass loss is expected to increase as the star ascends the AGB. Near the tip of the AGB, the mass loss rate can exceed $10^{-5} \mathbf{M}_{\circ}$ $\mathrm{yr}^{-1}$, or $1 \mathrm{M}_{\odot}$ can be lost in $10^{5} \mathrm{yr}$. Since the typical mass of PN is only $10^{-1} \mathrm{M}_{\circ}$, there is no doubt that mass loss on the AGB will have significant effect on the formation of PN (Kwok 1982). There has been increasing evidence that the remnant of the AGB wind is still present in PN. Large far-infrared continuum excesses have been observed in PN (Pottasch et al. 1984), with the amount of excess and the colour temperature both higher in young PN. Such excesses can be naturally explained by the dispersing remnant of the AGB envelope (Kwok 1990). The two most common dust constituents of AGB winds: silicate (in oxygen-rich stars) and SiC (in carbon stars) have been detected in PN (Aitkens \& Roche 1982; Zhang \& Kwok 1990). Molecular CO emission, which is a common property of AGB winds, is detected in many PN (Huggins \& Healy 1989). OH maser emission, which has been detected in over 1000 oxygen-rich AGB stars, have also been detected in several PN (Zijlstra et al. 1989). A scenario on how $\mathrm{OH}$ emission evolves from the AGB to PN has been outlined by Lewis (1989). High dynamic range CCD imaging of $\mathrm{PN}$ has revealed that most $\mathrm{PN}$ have faint outer haloes which are probably remnants of the AGB winds (Balick 1987).

Since up to several solar masses of material can be ejected during the AGB, there is more 
than enough material for the creation of a PN, and no hypothetical separate sudden ejection is needed. The question then becomes how this AGB wind material can be rearranged to look like a PN? It is clear that PN is not simply an ionized AGB wind. PN have higher densities and higher expansion velocities than AGB winds. The total amount of mass in the PN shell is small $\left(10^{-3}\right.$ to $\left.10^{-1} \mathrm{M}_{\sigma}\right)$ compared to the total amount ejected during the AGB. A separate mechanism is needed to accelerate and compress part of this previously ejected material into a PN shell.

\section{The interacting stellar winds model}

Let us suppose that AGB mass loss can continue until most of the hydrogen envelope is depleted by the wind process. When the envelope mass drops below $10^{-3} \mathrm{M}_{\odot}$ (depending on core mass, Schönberner 1983), the effective temperature of the star will increase. When the effective temperature exceeds a certain value $(\sim 5000 \mathrm{~K})$ the mass loss process can no longer continue. The small remaining hydrogen envelope will be consumed by shell burning. As the star evolves to higher temperature at constant luminosity (Paczyński 1971), the surface gravity (and escape velocity) of the star continues to increase. At a later point, a new wind with higher velocity is initiated. This new wind will sweep up the remnant of the AGB wind into a shell, producing a PN (Kwok, Purton \& FitzGerald 1978). The interactions of the new wind, the shell, and the AGB wind will lead to two shocks (Kahn 1983, Kwok 1983): an inner (probably adiabatic) shock that creates a high-temperature region which thermal pressure drives the expansion of the nebula; and an outer (isothermal) shock that compresses the AGB wind into a high density shell. These solutions lead to a uniformly expanding shell with mass and size linearly increasing with time (Volk \& Kwok 1985; Okorokov et al. 1985; Bedogni \& d'Ercole 1986; Schmidet-Voigt \& Köppen 1987a,b; Soker \& Livio 1989; Marten \& Schönberner 1991).

In addition to driving the dynamical evolution of PN, the new fast wind will also help to deplete the envelope mass of the star and hasten its evolution. Using the radiatively driven wind model of Pauldrach et al. (1988), the mass loss rate of this wind is lower than, but is still a significant fraction of, the nuclear burning rate. The fast wind therefore serves as the coupling agent between the nebular (dynamical) evolution and the central star evolution.

There is also a significant difference between whether the AGB is terminated by a wind, or by a sudden ejection. In a sudden ejection model, there is no guarantee how much mass will be left above the core. Even a small remaining amount will delay the evolution of the central star for thousands of years and the thus ionization of the nebula. However, if the AGB envelope is depleted by a gradual wind, we are assured that all that can be lost will be lost, and only the minimum remains.

\section{The proto-planetary nebula phase of evolution}

Since the far infrared emission observed in PN originates from the remnant of the AGB envelope, its low colour temperature can be understood as the result of the geometric dilution due to expansion. If this is the case, then objects in transition from AGB to PN can be discovered by searching for objects with intermediate infrared colours between AGB stars and PN. From the number of PN detected by the IRAS survey, it is estimated between 100-300 proto-planetary nebulae (PPN) should be in the IRAS

Point Source Catalog. Model calculations suggest that PPN should show a low temperature dust component as well as a reddened photosphere, with the latter increasing in strength as the envelope disperses (Bedijn 1987; Volk \& Kwok 1989). The search for PPN have been carried out by several groups (Parthasarathy \& Pottasch 1987; van der Veen 1988; Kwok, Hrivnak \& Volk 1990; Trams et al. 1991). As of 1991, over 20 PPN candidates have been identified. Optical spectroscopy of these objects show that most of them are F or G supergiants (Hrivnak, Kwok, \& Volk 1989). Many of these PPN candidates have been detected in $\mathrm{CO}$ and/or $\mathrm{OH}$ circumstellar emission, confirming that they are postAGB objects (Likkel 1989; Likkel et al. 1991; Woodsworth, Kwok, \& Chan 1990).

\section{Conclusion}

The evolutionary connection between the AGB and the PN evolutionary phases is now firmly established by infrared and molecular-line observations. Many properties of the circumstellar envelopes of AGB 
stars are detected in PN. While mass loss on the AGB has been confirmed as a necessary condition for the formation of PN, it is not a sufficient condition. Another phase of mass loss with a higher velocity is needed to compress and accelerate the remnant AGB envelope to the shell structure that is observed in PN. The discovery of PPN has also filled in an observational gap in the evolution between AGB and PN stages. However, a number of questions remain to be answered. Observations of PN haloes show that many have multi-shell structure (Chu et al. 1991) which could be related to the mass loss history on the AGB (see Wood, this volume). Many PN have "bipolar" morphology (Balick 1987), suggesting that mass loss on the AGB may not be spherically symmetric. Since the properties of PN is determined by both the dynamical evolution of the nebula and the evolution of the central star, a selfconsistent, coupled stellar-nebula model is necessary. On the theoretical side, the problem of the formation of PN demands a synthesis of stellar structure, stellar atmospheres, nebular physics and dynamics. On the observational side, it requires multi-wavelength observations in optical, infrared, millimetre, and radio wavelengths to obtain a comprehensive picture. For these reasons, the formation of PN truly qualifies as a modern astrophysical problem of unique richness.

\section{References}

Aitkens, D.K., \& Roche, P.F. 1982, MNRAS, 200, 217

Balick, B. 1987, $A J$, 94, 671

Bedijn, P.J. 1987, $A \& A, 186,136$

Bedogni, R., \& d'Ercole, A. 1986, A\&A, 157, 101

Chu, Y.H., Manchado, A., Jacoby, G.H., \& Kwitter, K.B. 1991, ApJ, 376, 150

Hrivnak, B.J., Kwok, S., \& Volk, K. 1989, ApJ, 346, 265.

Huggins, P.J., \& Healy, A.P. 1989, $A p J, 346,201$

Kahn, F.D., 1983, in LAU Symp 103: Planetary Nebulae, ed. D.R. Flower (Dordrecht:Reidel), p. 305

Kwok, S. 1982, ApJ, 258, 280

Kwok, S. 1983, in LAU Symp 103: Planetary Nebulae, ed. D.R. Flower (Dordrecht:Reidel), p. 293

Kwok, S. 1990, MNRAS, 244, 179

Kwok, S., Purton, C.R., \& FitzGerald, P.M. 1978, ApJ, 219, L125

Kwok, S., Hrivnak, B.J., \& Volk, K.M. 1990, in From Miras to Planetary Nebulae, ed. M.O. Mennessier, p. 435

Lewis, B.M. 1989, $A p J$, 338, 234

Likkel, L. 1989, $A p J, 344,350$

Likkel, L., Forveille, T., Omont, A., \& Morris, M. 1991, $A \& A, 246,153$

Marten, H., \& Schönberner, D. 1991, $A \& A$, in press

Okorokov, V.A., Shustov, B.M., Tutukov, A.V., \& Yorke, H.W. 1985, A\&A, 142, 441

Paczyński, B. 1971, Acta Astron, 31, 417

Pauldrach, A., Puls, J., Kudritzki, R.P., Méndez, R.H., Heap, S.R. 1988, A\&A, 207, 123

Parthasarathy, M. \& Pottasch, S.R. 1987, A\&A, 154, L16

Pottasch, S.R. et al. 1984, $A \& A, 138,10$

Renzini, A. 1982, in LAU Symp 103: Planetary Nebulae, ed. D.R. Flower (Dordrecht:Reidel), p.267

Schmidt-Voigt, M., \& Köppen, J. 1987a, $A \& A, 174,211$

Schmidt-Voigt, M., \& Köppen, J. 1987b, $A \& A, 174,223$

Schönberner, D. 1983, ApJ, 272, 708

Shklovskii, I. 1956, Astr. Zh., 33, 315

Soker, N., \& Livio, M. 1989, ApJ, 339, 268

Trams, N.R., Waters, L.B.F.M., Lamers, H.J.G.L.M, Waelkens, C., Geballe, T.R., \& Thé, P.S. 1991, $A \& A S, 87,361$

van der Veen, W.E.C.J. 1988, Thesis, Leiden Observatory, Netherlands

Volk, K., \& Kwok, S. 1985, $A \& A, 153,79$

Volk, K., \& Kwok, S. 1989, ApJ, 342, 345

Woodsworth, A.W., Kwok, S., \& Chan, S.J. 1990, $A \& A, 228,503$

Zhang, C.Y., \& Kwok, S. 1990, A\&A, 237, 479

Zijlstra, A.A., et al. 1989, $A \& A, 217,157$ 